\title{
Vectors for Zika virus may spread further than was previously thought, CDC reports
}

\author{
Michael McCarthy
}

Seattle

The combined range of the two mosquito species that transmit the Zika virus includes most of the eastern and central United States and extends as far north as Minnesota and Maine, a new assessment by the US Centers for Disease Control and Prevention (CDC) has found.

The primary Zika virus vector, the Aedes aegypti mosquito, tends to inhabit tropical and subtropical regions, but a second species that can also transmit the virus, Aedes albopictus, can survive in more temperate areas and has the potential to spread further north, as shown on maps released by the CDC on 30 March. ${ }^{1}$ Earlier assessments had indicated that these species were primarily found in the south.

To date, no cases of locally acquired mosquito-borne infections in the continental US have been reported, and the CDC noted that the presence of these mosquitoes does not indicate a high transmission risk if the virus enters these regions. Other factors determine whether the virus will be spread in a particular locality, the CDC said, such as the number of mosquitoes in an area, their feeding habits, proximity to people, and ability to transmit the virus.

Aedes aegypti mosquitoes, for example, are more likely to spread the virus because they tend to live near to, and prefer to feed on, people and are more efficient virus transmitters. Aedes albopictus, however, feed on animals as well as people and are less efficient transmitters.

The CDC said that it cannot predict whether Zika virus will spread to the continental US but that areas that have experienced outbreaks of chikungunya and dengue-infections caused by related mosquito-borne viruses_-are considered at higher risk.
These areas include parts of Hawaii, Florida, and Texas, three states that have had local outbreaks of chikungunya and dengue. By 30 March, 312 Zika cases in the US had been reported to the CDC. All cases have been associated with travel to areas where the virus has been actively transmitted. Of these, 27 were pregnant women and six involved sexual contact with a partner who had traveled to regions where the virus was actively circulating. In the US territories of Puerto Rico, US Virgin Islands, and American Samoa, however, 352 cases of Zika have been reported, 349 of which were locally acquired and only three of which were associated with travel.

Meanwhile, researchers at Purdue University in the US have worked out the Zika virus's structure, a breakthrough that they hope will lead to treatment and vaccine development. ${ }^{2}$

Richard Kuhn, an author of the Purdue study and director of the Purdue Institute for Inflammation, Immunology, and Infectious Diseases, said that working out the structure advances the understanding of Zika, a virus about which little is known.

Kuhn said, "The structure of the virus provides a map that shows potential regions of the virus that could be targeted by a therapeutic treatment, used to create an effective vaccine or to improve our ability to diagnose and distinguish Zika infection from that of other related viruses."

1 Centers for Disease Control and Prevention. Estimated range of Aedes albopictus and Aedes aegypti in the United States, 2016. Mar 2016. www.cdc.gov/zika/pdfs/zika-mosquitomaps.pdf.

Sirohi D, Chen Z, Sun L, et al. The 3.8 Å resolution cryo-EM structure of Zika virus. Science 2016; published online 31 Mar. doi:10.1126/science.aaf5316.

Published by the BMJ Publishing Group Limited. For permission to use (where not already granted under a licence) please go to http://group.bmj.com/group/rights-licensing/ permissions 